\title{
Exploring $s d$-shell nuclei from two- and three-nucleon interactions with realistic saturation properties
}

\author{
J. Simonis, ${ }^{1,2, *}$ K. Hebeler, ${ }^{1,2, \dagger}$ J. D. Holt,${ }^{3, \ddagger}$ J. Menéndez, ${ }^{4,}{ }^{\S}$ and A. Schwenk ${ }^{1,2,}$ ฯ \\ ${ }^{1}$ Institut für Kernphysik, Technische Universität Darmstadt, 64289 Darmstadt, Germany \\ ${ }^{2}$ ExtreMe Matter Institute EMMI, GSI Helmholtzzentrum für Schwerionenforschung GmbH, 64291 Darmstadt, Germany \\ ${ }^{3}$ TRIUMF, 4004 Wesbrook Mall, Vancouver, British Columbia, V6T 2 A3 Canada \\ ${ }^{4}$ Department of Physics, University of Tokyo, Hongo, Tokyo 113-0033, Japan
}

\begin{abstract}
We study ground- and excited-state properties of all $s d$-shell nuclei with neutron and proton numbers $8 \leqslant N, Z \leqslant 20$, based on a set of low-resolution two- and three-nucleon interactions that predict realistic saturation properties of nuclear matter. We focus on estimating the theoretical uncertainties due to variation of the resolution scale, the low-energy couplings, as well as from the many-body method. The experimental two-neutron and two-proton separation energies are reasonably well reproduced, with an uncertainty range of $\sim 5 \mathrm{MeV}$. The first excited $2^{+}$energies also show overall agreement, with a more narrow uncertainty range of $\sim 500 \mathrm{keV}$. In most cases, this range is dominated by the uncertainties in the Hamiltonian.
\end{abstract}

PACS numbers: 21.10.-k, 21.30.-x, 21.60.Cs, 27.30.+t

Introduction. Recent advances in nuclear theory have established the importance of three-nucleon $(3 \mathrm{~N})$ forces in understanding the structure of medium-mass nuclei, for the evolution to the neutron and proton driplines [1$5]$ and the formation of shell structure [6-13]. Threenucleon forces are also key for realistic saturation properties of nuclear matter [14-18], which in turn are obtained from global analyses of all nuclei. To date, ab initio studies of medium-mass nuclei have largely focused on closedshell nuclei or isotopic chains, generally in the vicinity of semi-magic nuclei, and no comprehensive study exists to explore nuclear forces over a full range of the nuclear chart, such as the $s d$ shell.

An additional challenge is the quantification of theoretical uncertainties [19]. Calculations in oxygen and calcium isotopes based on nuclear forces derived from chiral effective field theory (EFT) $[20,21]$ suggest that the uncertainties from the many-body methods are well controlled $[4,5,11,13,22]$. Therefore, uncertainties in the input Hamiltonian, such as truncations in the chiral EFT expansion or uncertainties in the low-energy couplings, likely remain the dominant source of uncertainty. Note that recently, first studies of the statistical uncertainties from numerically optimized chiral forces $[23,24]$ and to quantify correlations between chiral EFT couplings $[25,26]$ have been performed.

In this work we investigate all $s d$-shell nuclei based on chiral two-nucleon (NN) and 3N interactions with realistic saturation properties. We derive microscopic valence-space Hamiltonians, which we diagonalize to obtain ground-state energies, two-neutron and two-proton separation energies, and first excited $2_{1}^{+}$energies. By varying the resolution scale in nuclear forces and the lowenergy $3 \mathrm{~N}$ couplings, we provide theoretical uncertainty estimates for these observables. In addition we also explore the uncertainty associated due to the many-body calculations. We find that the resulting energy ranges, which are in good agreement with experimental data throughout the region, are mainly driven by uncertainties in the Hamiltonian.

Nuclear interactions. At the NN level, we start from the next-to-next-to-next-to-leading order $\left(\mathrm{N}^{3} \mathrm{LO}\right)$ $500 \mathrm{MeV}$ potential of Entem and Machleidt (EM) [27]. We then use the similarity renormalization group (SRG) $[28,29]$ to evolve this interaction to a series of lowresolution scales $\lambda_{\mathrm{NN}}=1.8,2.0,2.2 \mathrm{fm}^{-1}$. Taking chiral EFT as a general low-momentum basis, we combine each SRG-evolved NN interaction with the leading $\mathrm{N}^{2} \mathrm{LO} 3 \mathrm{~N}$ forces $[30,31]$, where the $c_{i}$ couplings in the two-pionexchange $3 \mathrm{~N}$ interaction are taken consistently with the $\mathrm{NN}$ interaction: $c_{1}=-0.81 \mathrm{GeV}^{-1}, c_{3}=-3.2 \mathrm{GeV}^{-1}$, $c_{4}=5.4 \mathrm{GeV}^{-1}$. We also vary independently the $3 \mathrm{~N}$ cutoff $\Lambda_{3 \mathrm{~N}}=2.0,2.5 \mathrm{fm}^{-1}$ for the $\lambda_{\mathrm{NN}}=2.0 \mathrm{fm}^{-1}$ interaction. In addition, to probe uncertainties in the $c_{i}$ couplings, we use $3 \mathrm{~N}$ forces with the $c_{i}$ values obtained from the Nijmegen NN partial wave analysis (PWA): $c_{1}=$ $-0.76 \mathrm{GeV}^{-1}, c_{3}=-4.78 \mathrm{GeV}^{-1}, c_{4}=3.96 \mathrm{GeV}^{-1}[32]$ for the $\lambda_{\mathrm{NN}} / \Lambda_{3 \mathrm{~N}}=2.0 / 2.0 \mathrm{fm}^{-1}$ interaction. For all Hamiltonians, the low-energy couplings $c_{D}, c_{E}$ in the $3 \mathrm{~N}$ one-pion-exchange and $3 \mathrm{~N}$ contact interaction have been fit to the ${ }^{3} \mathrm{H}$ binding energy and ${ }^{4} \mathrm{He}$ charge radius using Faddeev- and Fadeev-Yakubowsky calculations [15]. With this set of five $\mathrm{NN}+3 \mathrm{~N}$ interactions, which predict nuclear saturation properties within uncertainties [15], we explore all $s d$-shell nuclei. The different interactions are denoted as 1.8/2.0 (EM), 2.0/2.0 (EM), 2.2/2.0 (EM), $2.0 / 2.5(\mathrm{EM}), 2.0 / 2.0 \mathrm{fm}^{-1}$ (EM+PWA), where the labeling indicates $\lambda_{\mathrm{NN}} / \Lambda_{3 \mathrm{~N}}$ and the $c_{i}$ couplings used.

Microscopic valence-space Hamiltonians. Based on the above interactions, we construct an effective valencespace Hamiltonian, $H_{\text {eff }}=\sum_{i} \varepsilon_{i} a_{i}^{\dagger} a_{i}+V_{\text {eff }}$, where $\varepsilon_{i}$ denote the single-particle energies (SPEs), and $V_{\text {eff }}$ is the effective two-body interaction for valence nucleons. With $H_{\text {eff }}$ we perform valence shell-model calculations, where 
the many-body problem is solved exactly for the particles in the valence space on top of a closed core.

Recently, nonperturbative methods for calculating $H_{\text {eff }}$ in medium-mass nuclei have been developed [33-36] and applied to the oxygen and very recently fluorine [37] isotopes. In this work, we use many-body perturbation theory (MBPT) [38], which provides a diagrammatic orderby-order expansion for SPEs and $V_{\text {eff }}$, to take into account many-body processes outside the valence space. At third order, MBPT based on RG/SRG-evolved interactions with low cutoffs shows a reasonable order-byorder convergence for SPEs and $V_{\text {eff }}$ in medium-mass nuclei [39]. To explore uncertainties associated with MBPT, we study valence-shell Hamiltonians obtained at secondand third-order MBPT. In contrast to phenomenological interactions, such as USDA/B [40] that fit both SPEs and $V_{\text {eff }}$ to experimental $s d$-shell data, our results are without adjustments. Therefore, we do not expect to reach accuracies comparable to the best fit USD interactions.

Studies of oxygen $[41,42]$ and calcium $[8,10,39]$ isotopes showed that extending the valence space beyond one major shell provides additional binding and can lead to improvements for neutron-rich systems. However since our main interest is to perform a comprehensive study of $s d$-shell nuclei, estimating the theoretical uncertainty associated with the initial Hamiltonians, we limit our valence space to the $s d$ shell $\left(d_{5 / 2}, d_{3 / 2}\right.$, and $s_{1 / 2}$ proton and neutron single-particle orbitals on top of a ${ }^{16} \mathrm{O}$ core). We work in a harmonic-oscillator basis with $\hbar \omega=13.53 \mathrm{MeV}$, appropriate for the $s d$ shell, and scale all matrix elements of $V_{\text {eff }}$ and bound SPEs by $A^{-1 / 3}$ to correct for the increase in nuclear size. For all $\lambda_{\mathrm{NN}}$ considered, the calculations are converged in a basis consisting of 13 major shells for NN forces [39]. For 3N forces, we allow a total energy of the three single-particle states up to $12 \hbar \omega$ $\left(E_{3 \max }=12\right)$ in a basis of 13 major shells.

Results. Figure 1 shows the ground-state energies of magnesium and chlorine isotopes compared to the Atomic Mass Evaluation (AME 2012) [43]. The secondand third-order MBPT results are represented by the blue, darker and the cyan, lighter bands, respectively, where the width of each band is spanned by the five different $\mathrm{NN}+3 \mathrm{~N}$ interactions considered.

The experimental ground-state energies for magnesium isotopes are generally within our uncertainty band, with neutron-rich isotopes at the lower side. Only the most neutron-rich isotopes are underbound in our calculations. On the other hand, the ground-state energies of all chlorine isotopes are in good agreement with our uncertainty band, and they are typically within the lower side of the the third-order MBPT band, defined by the $\lambda_{\mathrm{NN}} / \Lambda_{3 \mathrm{~N}}=1.8 / 2.0,2.0 / 2.0,2.2 / 2.0,2.0 / 2.5 \mathrm{fm}^{-1}$ interactions. In general we find better agreement between our results and experiment for the isotopic chains of heavier elements, which suggests a somewhat too weak neutronneutron interaction in our $s d$-shell calculations. This was

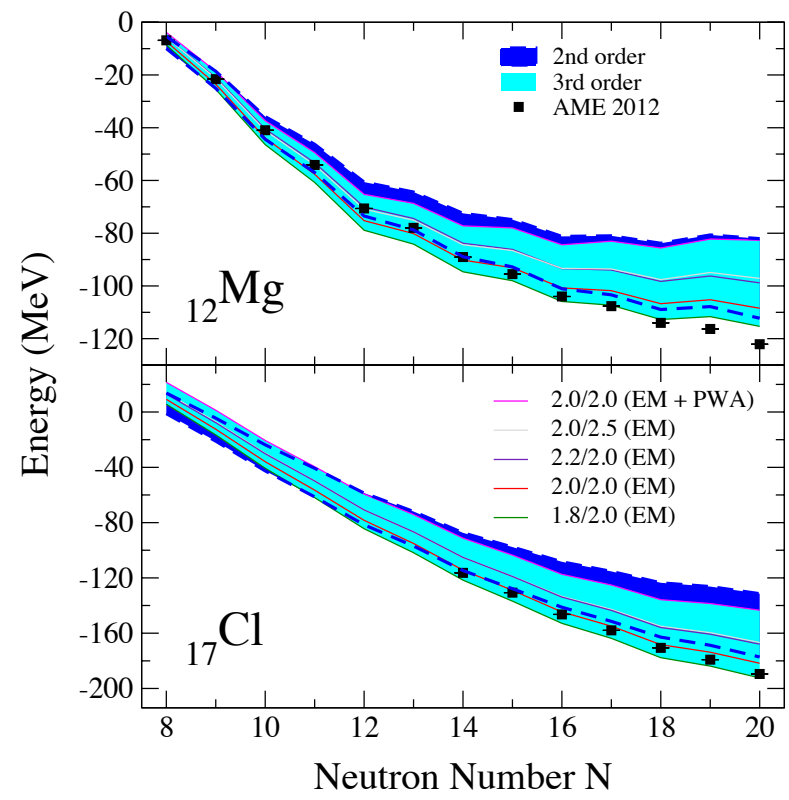

FIG. 1. Ground-state energies of the magnesium (top) and chlorine isotopes (bottom panel) relative to ${ }^{16} \mathrm{O}$ at second (blue, darker band) and third order (cyan, lighter band) in MBPT and compared to the Atomic Mass Evaluation (AME 2012) [43]. The uncertainty bands are spanned by the five different $\mathrm{NN}+3 \mathrm{~N}$ interactions (see text for details). The ordering in the legend is with decreasing ground-state energies.

also observed in Refs. [41, 42] for the oxygen isotopes.

The estimated uncertainties in calculated ground-state energies are dominated by the different input Hamiltonians. Specifically, the resolution-scale dependence by varying $\lambda_{\mathrm{NN}}$ from $1.8-2.2 \mathrm{fm}^{-1}$ with $\Lambda_{3 \mathrm{~N}}=2.0 \mathrm{fm}^{-1}$ is somewhat larger than the $\Lambda_{3 \mathrm{~N}}$ dependence from $2.0-$ $2.5 \mathrm{fm}^{-1}$ for $\lambda_{\mathrm{NN}}=2.0 \mathrm{fm}^{-1}$. This results in a combined resolution-scale dependence of approximately $1.0 \mathrm{MeV}$ per valence particle in ${ }^{32} \mathrm{Mg}$ and ${ }^{37} \mathrm{Cl}$. When also including the PWA $c_{i}$ values in $3 \mathrm{~N}$ forces, the uncertainty roughly doubles to about $2.0 \mathrm{MeV}$ per valence particle. For both second- and third-order MBPT bands, the $2.0 / 2.0 \mathrm{fm}^{-1}$ (EM+PWA) interactions generally define the least bound calculations (for ground-state energies, the upper end of the bands).

The difference between second- and third-order MBPT results is relatively small compared to the width of each band, indicating a reasonable, but still incomplete, convergence of the MBPT approach in this region. For magnesium and chlorine, third-order results are more bound because of more attractive proton-neutron interactions, whereas for oxygen (not shown), second-order results are more bound than at third order mainly due to the neutron single-particle energies. When the uncertainty associated to the MBPT is also included, the total uncertainty increases to $2.1 \mathrm{MeV}$ and $2.8 \mathrm{MeV}$ per valence particle in ${ }^{32} \mathrm{Mg}$ and ${ }^{37} \mathrm{Cl}$, respectively. 


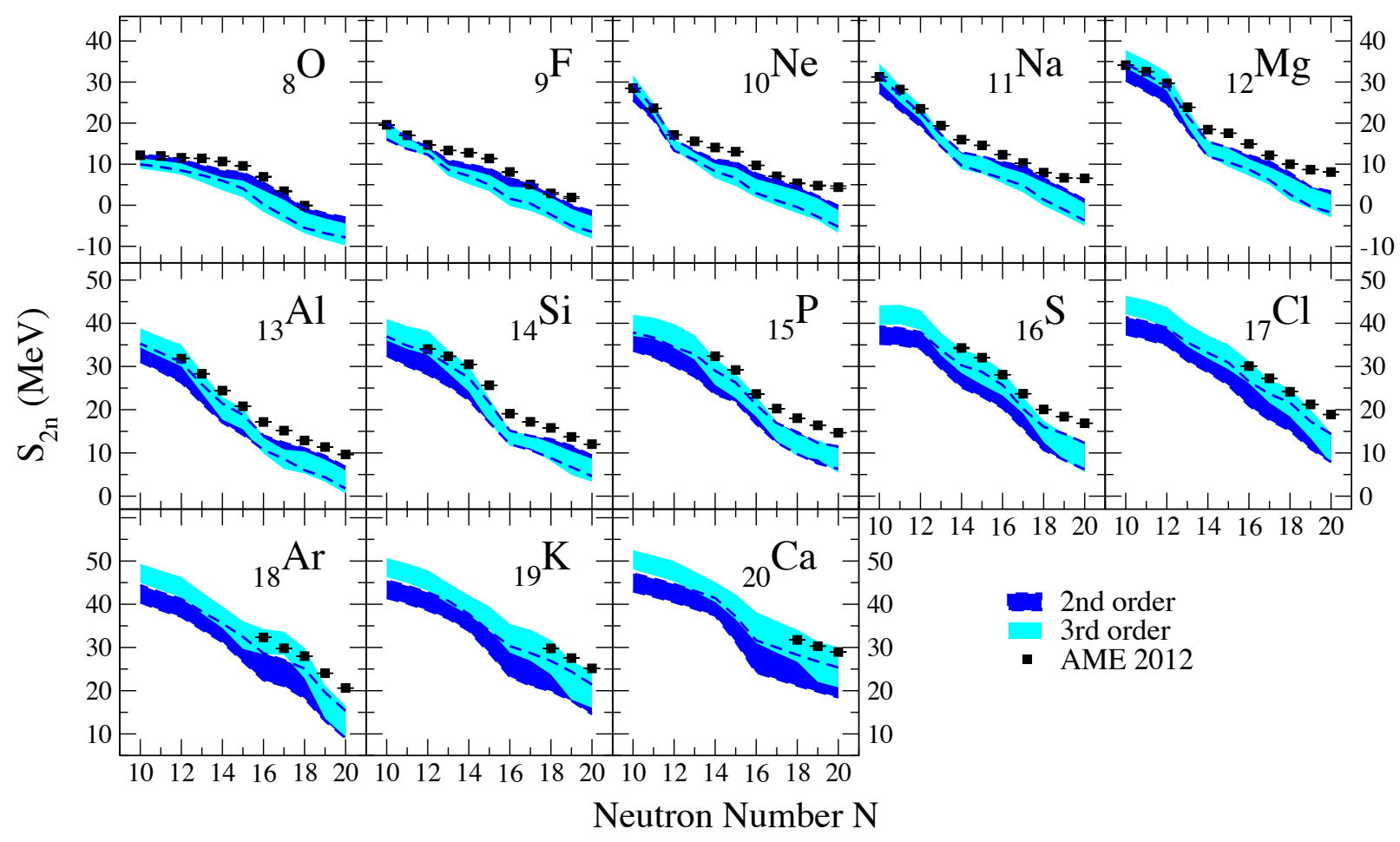

FIG. 2. Uncertainty estimates for the two-neutron separation energies $S_{2 \mathrm{n}}$ of $s d$-shell isotopic chains at second (blue, darker band) and third order (cyan, lighter band) in MBPT and compared to the Atomic Mass Evaluation (AME 2012) [43].

Figure 2 compares theoretical and experimental twoneutron separation energies $S_{2 \mathrm{n}}$ for all isotopic chains from oxygen to calcium $(Z=8-20)$. The theoretical calculations describe the overall experimental trends reasonably well, but in general our uncertainty bands underestimate the empirical values. This is especially the case in lighter elements and for the most neutron-rich nuclei for all isotopic chains. This is probably related to the underbinding of the $s d$-shell calculations when valence neutron-neutron interactions are dominant. We also note that around $N=20$, the ground states of ${ }^{29,30} \mathrm{Ne}$ [44, 45], ${ }^{30,31} \mathrm{Na}[46,47]$, and ${ }^{31,32} \mathrm{Mg}[48,49]$ are dominated by deformed configurations not captured in our $s d$-shell calculations (this is the so-called island of inversion). Consequently, our bands do not reproduce the change in slope of $S_{2 \mathrm{n}}$ around $N=20$ for $\mathrm{Ne}, \mathrm{Na}$, or $\mathrm{Mg}$.

Similar to the ground-state energies, the dominant uncertainties arise from the different Hamiltonians, with smaller differences between second- and third-order MBPT results. Typical the uncertainty range for $S_{2 \mathrm{n}}$ is $\sim$ $5 \mathrm{MeV}$. The exception are $N<Z$ isotopes, more visible in heavier elements, where the difference between secondand third-order results is comparable to the uncertainty between input Hamiltonians, due to too weak protonneutron interactions at second-order MBPT, adding up to a total uncertainty of $\sim 10 \mathrm{MeV}$.

In Fig. 3 we show the two-proton separation energy $S_{2 \mathrm{p}}$ for all isotonic chains from $N=8$ to $N=20$. Our re- sults agree very well with experiment in all cases, and remarkably most experimental values fall within the thirdorder MBPT band. Only in few proton-deficient and very proton-rich nuclei do experimental $S_{2 \mathrm{p}}$ lie within the second-order band. Since there are fewer proton-rich nuclei known experimentally than neutron-rich nuclei, $S_{2 \mathrm{p}}$ are in general informative about proton-neutron interactions. The much better agreement in $S_{2 \mathrm{p}}$ than for $S_{2 \mathrm{n}}$ compared to experiment suggest that the different Hamiltonians considered capture better (mostly isoscalar) proton-neutron interactions than neutron-neutron interactions. Again, the sensitivity to the input Hamiltonians dominates the theoretical $S_{2 \mathrm{p}}$ uncertainties (with a similar range of $\sim 5 \mathrm{MeV}$ ), except for proton-deficient nuclei where the MBPT uncertainty is comparable (with a total uncertainty of $\sim 10 \mathrm{MeV}$ ).

Finally in Fig. 4, the calculated first excited $2_{1}^{+}$energies are compared to experimental data for all eveneven $s d$-shell isotopes. The spread of the uncertainty band is typically smaller than $\sim 500 \mathrm{keV}$, with generally reasonable agreement to experiment. However, in the cases with high-lying $2_{1}^{+}$states, indicative of shell closures $\left({ }^{22} \mathrm{O},{ }^{24} \mathrm{O},{ }^{22} \mathrm{Si},{ }^{34} \mathrm{Si},{ }^{34} \mathrm{Ca}\right)$, the uncertainty can be as large as $\sim 1 \mathrm{MeV}$. This means that, while our bands in general predict shell closures consistently, the actual excitation of the $2_{1}^{+}$state is very sensitive to the details of the input Hamiltonian. The width of the uncertainty band is mostly due to the $2.0 / 2.0 \mathrm{fm}^{-1}$ (EM+PWA) interaction, 


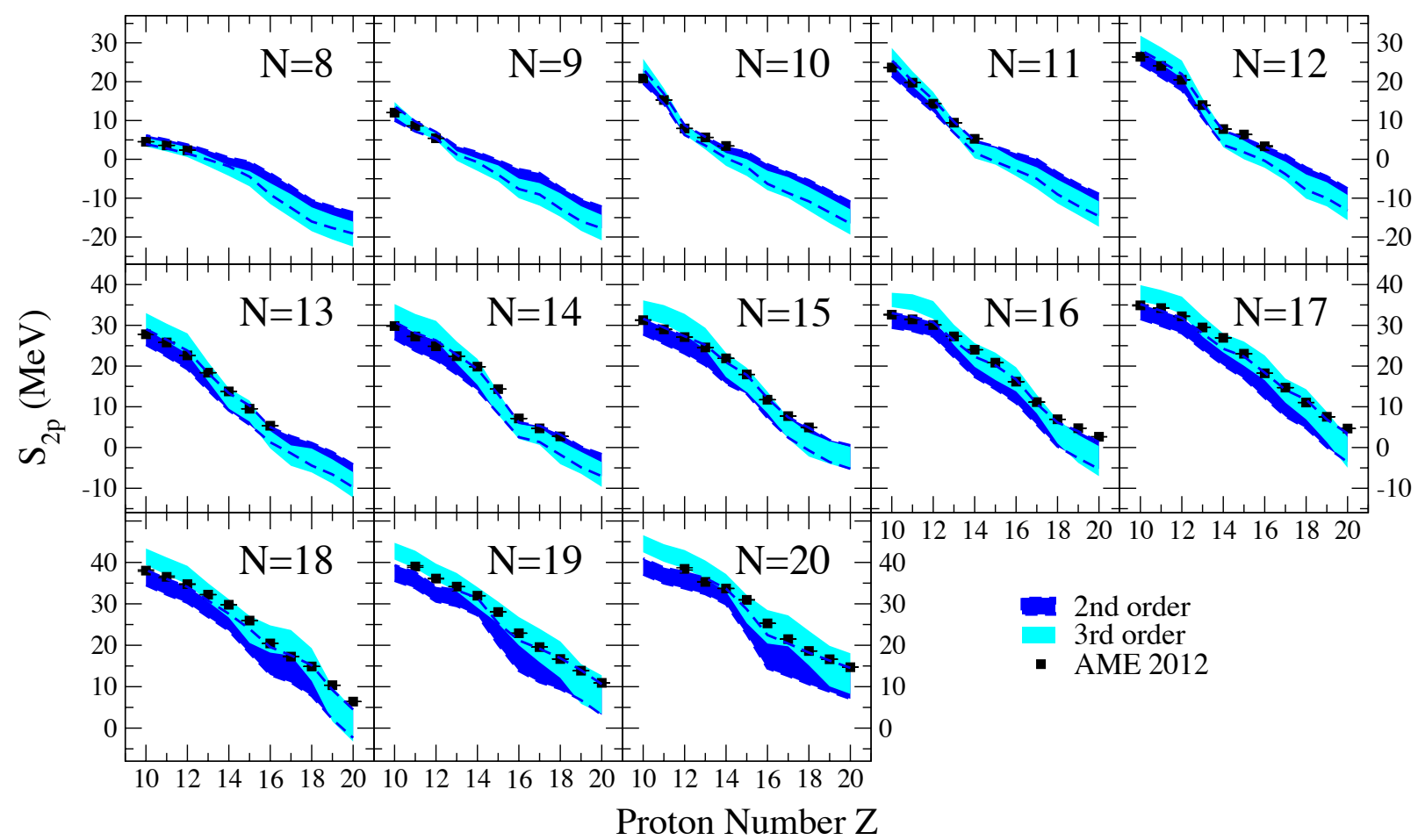

FIG. 3. Uncertainty estimates for two-proton separation energies $S_{2 \mathrm{p}}$ of $s d$-shell isotonic chains at second (blue, darker band) and third order (cyan, lighter band) in MBPT and compared to the Atomic Mass Evaluation (AME 2012) [43].

which is also responsible for the unusually large uncertainty band in ${ }^{36} \mathrm{Ar}$. In general, the second- and thirdorder MBPT bands mostly overlap, except for $N \sim Z$ argon and calcium isotopes, where only third-order MBPT results are in agreement to experiment. Similar to the case of $S_{2 \mathrm{n}}$, we also note that the $2_{1}^{+}$states within the island of inversion, the $N=20$ isotopes ${ }^{30} \mathrm{Ne}$ [45] and ${ }^{32} \mathrm{Mg}$ [49], are deformed, and their relatively low excitation energies cannot be well described in our $s d$-shell calculations.

Summary. We have presented a comprehensive study of ground-state energies, $S_{2 \mathrm{n}}, S_{2 \mathrm{p}}$, and first excited $2_{1}^{+}$energies for all $s d$-shell nuclei: isotopic chains from oxygen to calcium and isotonic chains from $N=8$ to $N=20$. This is based on $\mathrm{NN}+3 \mathrm{~N}$ Hamiltonians that have been fitted only to $A=3,4$ nuclei that predict realistic saturation properties of nuclear matter, without additional adjustments. We have focused on estimating the theoretical uncertainties due to the different input Hamiltonians and associated with the many-body calculations. We find reasonable agreement to experimental data, especially in nuclei dominated by valence proton-neutron interactions. For neutron-rich systems, calculations in extended valence spaces are needed, due to too weak neutron-neutron interactions in the $s d$ shell.

Generally, we find that the estimated theoretical uncertainties are dominated by differences in the $\mathrm{NN}+3 \mathrm{~N}$ interactions. While the present first study is limited to the EM $500 \mathrm{MeV} \mathrm{N}{ }^{3} \mathrm{LO} \mathrm{NN}$ potential, it will be important to perform more comprehensive studies. These efforts will incorporate the exploration of different fitting procedures for the $3 \mathrm{~N}$ low-energy constants $c_{D}, c_{E}$, different regulator forms and cutoff values for $\mathrm{NN}$ and $3 \mathrm{~N}$ interactions as well as order-by-order convergence studies in the chiral EFT expansion [51-54]. For improved studies of the resolution scale dependence, we plan to perform calculations based on consistently SRG-evolved $\mathrm{NN}$ and $3 \mathrm{~N}$ interactions $[55,56]$. Improving our uncertainty estimates due the many-body calculation is more challenging, because corrections to MBPT (i.e., fourthorder contributions) are not attainable at present. However, nonperturbative methods for valence-space Hamiltonians can provide a controlled framework to assess the many-body approximations [33-36].

We thank H. Hergert, N. Tsunoda, and T. Otsuka for very useful discussions. This work was supported by the ERC Grant No. 307986 STRONGINT, the BMBF under Contract No. 05P15RDFN1, the National Research Council of Canada and NSERC, by an International Research Fellowship from the Japan Society for the Promotion of Science (JSPS), and JSPS KAKENHI grant No. $26 \cdot 04323$. Computations were performed with an allocation of computing resources at the Jülich Supercomputing Center. 


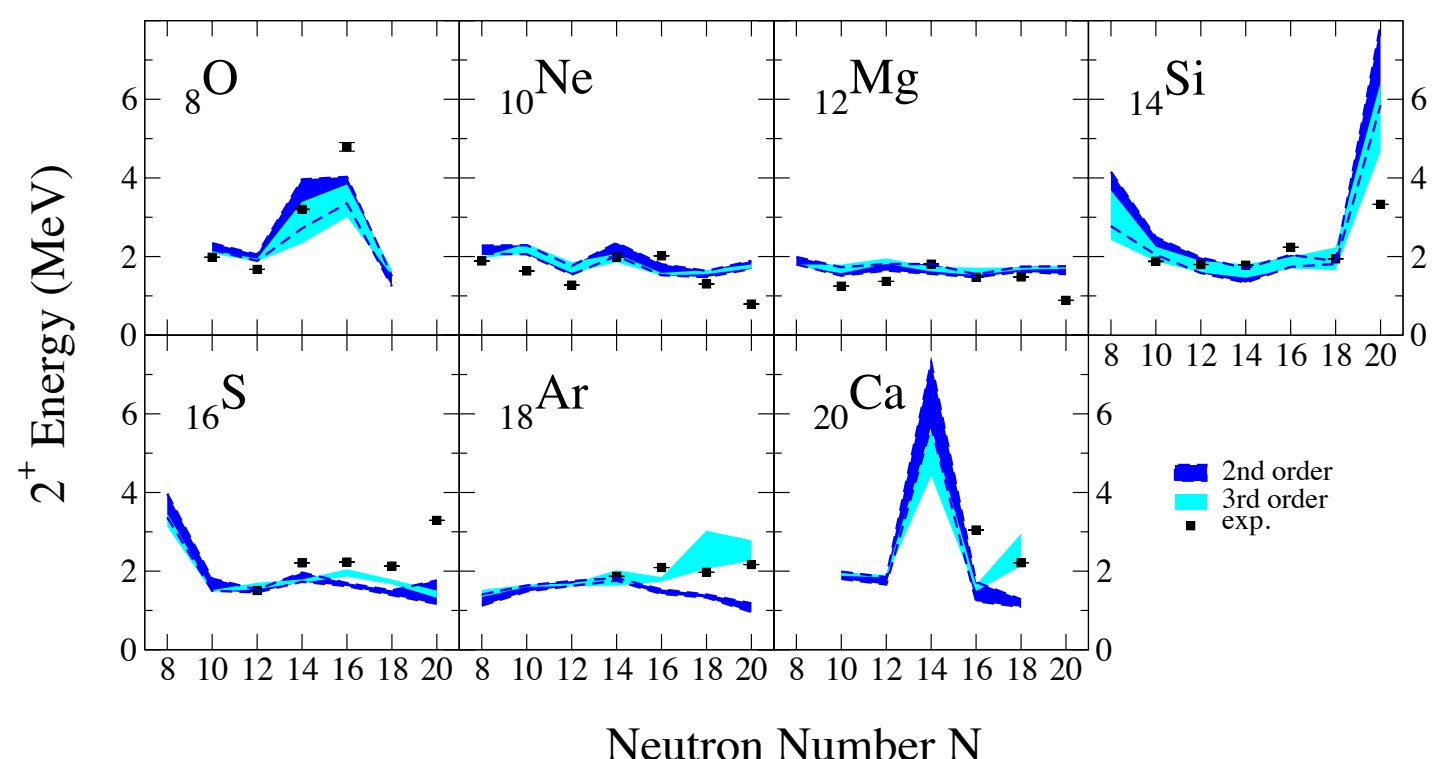

FIG. 4. Uncertainty estimates for excitation energies of the first $2_{1}^{+}$states in even-even $s d$-shell isotopes at second (blue, darker band) and third order (cyan, lighter band) and compared to experimental data from ENSDF [50].

* E-mail: simonis@theorie.ikp.physik.tu-darmstadt.de

† E-mail: kai.hebeler@physik.tu-darmstadt.de

¥ E-mail: jholt@triumf.ca

$\S$ E-mail: menendez@nt.phys.s.u-tokyo.ac.jp

ฯ E-mail: schwenk@physik.tu-darmstadt.de

[1] T. Otsuka, T. Suzuki, J. D. Holt, A. Schwenk, and Y. Akaishi, Phys. Rev. Lett. 105, 032501 (2010).

[2] G. Hagen, M. Hjorth-Jensen, G. R. Jansen, R. Machleidt, and T. Papenbrock, Phys. Rev. Lett. 108, 242501 (2012).

[3] J. D. Holt, J. Menéndez, and A. Schwenk, Phys. Rev. Lett. 110, 022502 (2013).

[4] A. Cipollone, C. Barbieri, and P. Navrátil, Phys. Rev. Lett. 111, 062501 (2013).

[5] H. Hergert, S. Binder, A. Calci, J. Langhammer, and R. Roth, Phys. Rev. Lett. 110, 242501 (2013).

[6] J. D. Holt, T. Otsuka, A. Schwenk, and T. Suzuki, J. Phys. G 39, 085111 (2012).

[7] G. Hagen, M. Hjorth-Jensen, G. R. Jansen, R. Machleidt, and T. Papenbrock, Phys. Rev. Lett. 109, 032502 (2012).

[8] A. T. Gallant et al., Phys. Rev. Lett. 109, 032506 (2012).

[9] J. D. Holt, J. Menéndez, and A. Schwenk, J. Phys. G 40, 075105 (2013).

[10] F. Wienholtz et al., Nature 498, 346 (2013).

[11] V. Somà, A. Cipollone, C. Barbieri, P. Navrátil, and T. Duguet, Phys. Rev. C 89, 061301(R) (2014).

[12] S. Binder, J. Langhammer, A. Calci, and R. Roth, Phys. Lett. B 736, 119 (2014).

[13] H. Hergert, S. K. Bogner, T. D. Morris, S. Binder, A. Calci, et al., Phys. Rev. C 90, 041302(R) (2014).

[14] S. K. Bogner, A. Schwenk, R. J. Furnstahl, and A. Nogga, Nucl. Phys. A 763, 59 (2005).

[15] K. Hebeler, S. K. Bogner, R. J. Furnstahl, A. Nogga, and A. Schwenk, Phys. Rev. C 83, 031301(R) (2011).

[16] G. Hagen, T. Papenbrock, A. Ekström, K. Wendt,
G. Baardsen, et al., Phys. Rev. C 89, 014319 (2014).

[17] A. Carbone, A. Rios, and A. Polls, Phys. Rev. C 88, 044302 (2013).

[18] L. Coraggio, J. W. Holt, N. Itaco, R. Machleidt, L. E. Marcucci, and F. Sammarruca, Phys. Rev. C 89, 044321 (2014).

[19] J. Dobaczewski, W. Nazarewicz, and P.-G. Reinhard, J. Phys. G 41, 074001 (2014).

[20] E. Epelbaum, H.-W. Hammer, and U.-G. Meißner, Rev. Mod. Phys. 81, 1773 (2009).

[21] R. Machleidt and D. R. Entem, Phys. Rept. 503, 1 (2011).

[22] G. Hagen, T. Papenbrock, M. Hjorth-Jensen, and D. J. Dean, Rept. Prog. Phys. 77, 096302 (2014).

[23] A. Ekström, G. Baardsen, C. Forssén, G. Hagen, M. Hjorth-Jensen, et al., Phys. Rev. Lett. 110, 192502 (2013).

[24] A. Ekström, G. R. Jansen, K. A. Wendt, G. Hagen, T. Papenbrock, et al., Phys. Rev. C 91, 051301(R) (2015).

[25] A. Ekström, B. D. Carlsson, K. A. Wendt, C. Forssén, M. Hjorth-Jensen, R. Machleidt, and S. M. Wild, J. Phys. G 42, 034003 (2015).

[26] B. D. Carlsson, A. Ekström, C. Forssén, D. F. Strömberg, O. Lilja, M. Lindby, B. A. Mattsson, and K. A. Wendt, arXiv:1506.02466.

[27] D. R. Entem and R. Machleidt, Phys. Rev. C 68, 041001(R) (2003).

[28] S. K. Bogner, R. J. Furnstahl, and R. J. Perry, Phys. Rev. C 75, 061001(R) (2007).

[29] S. K. Bogner, R. J. Furnstahl, and A. Schwenk, Prog. Part. Nucl. Phys. 65, 94 (2010).

[30] U. van Kolck, Phys. Rev. C 49, 2932 (1994).

[31] E. Epelbaum, A. Nogga, W. Glöckle, H. Kamada, U.G. Meißner, and H. Witała, Phys. Rev. C 66, 064001 (2002).

[32] M. C. M. Rentmeester, R. G. E. Timmermans, and J. J. 
de Swart, Phys. Rev. C 67, 044001 (2003).

[33] K. Tsukiyama, S. K. Bogner, and A. Schwenk, Phys. Rev. C 85, 061304(R) (2012).

[34] S. K. Bogner, H. Hergert, J. D. Holt, A. Schwenk, S. Binder, et al., Phys. Rev. Lett. 113, 142501 (2014).

[35] G. R. Jansen, J. Engel, G. Hagen, P. Navratil, and A. Signoracci, Phys. Rev. Lett. 113, 142502 (2014).

[36] E. Dikmen, A. F. Lisetski, B. R. Barrett, P. Maris, A. M. Shirokov, and J. P. Vary, Phys. Rev. C 91, 064301 (2015).

[37] L. Cáceres et al., Phys. Rev. C 92, 014327 (2015).

[38] M. Hjorth-Jensen, T. T. S. Kuo, and E. Osnes, Phys. Rept. 261, 125 (1995).

[39] J. D. Holt, J. Menéndez, J. Simonis, and A. Schwenk, Phys. Rev. C 90, 024312 (2014).

[40] B. A. Brown and W. A. Richter, Phys. Rev. C 74, 034315 (2006).

[41] J. D. Holt, J. Menéndez, and A. Schwenk, Eur. Phys. J. A 49, 39 (2013).

[42] C. Caesar et al. (R3B collaboration), Phys. Rev. C 88, 034313 (2013).

[43] M. Wang, G. Audi, A. H. Wapstra, F. G. Kondev,
M. MacCormick, X. Xu, and B. Pfeiffer, Chin. Phys. C 36, 1603 (2012).

[44] M. Belleguic et al., Phys. Rev. C 72, 054316 (2005).

[45] Y. Yanagisawa et al., Phys. Lett. B 566, 84 (2003).

[46] V. Tripathi et al., Phys. Rev. C 76, 021301(R) (2007).

[47] P. Doornenbal et al., Phys. Rev. C 81, 041305(R) (2010).

[48] M. Seidlitz et al., Phys. Lett. B 700, 181 (2011).

[49] T. Motobayashi et al., Phys. Lett. B 346, 9 (1995).

[50] http://www.nndc.bnl.gov/ensdf/.

[51] E. Epelbaum, H. Krebs, and U.-G. Meißner, Eur. Phys. J. A 51, 53 (2015).

[52] E. Epelbaum, H. Krebs, and U.-G. Meißner, arXiv:1412.4623.

[53] K. Hebeler, H. Krebs, E. Epelbaum, J. Golak, and R. Skibinski, Phys. Rev. C 91, 044001 (2015).

[54] R. J. Furnstahl, N. Klco, D. R. Phillips, and S. Wesolowski, arXiv:1506.01343.

[55] E. D. Jurgenson, P. Navrátil, and R. J. Furnstahl, Phys. Rev. Lett. 103, 082501 (2009).

[56] K. Hebeler, Phys. Rev. C 85, 021002(R) (2012). 